\title{
BUCKLING OF PERFORATED PLATES USING THE DUAL RECIPROCITY BOUNDARY ELEMENT METHOD
}

\author{
ROMILDO APARECIDO SOARES JR ${ }^{1}$, LEANDRO PALERMO JR ${ }^{1} \&$ LUIZ CARLOS WROBEL ${ }^{2,3}$ \\ ${ }^{1}$ School of Civil Engineering, Architecture and Urban Design, State University of Campinas, Brazil \\ ${ }^{2}$ Department of Mechanical and Aerospace Engineering, Brunel University London, UK \\ ${ }^{3}$ Department of Civil and Environmental Engineering, Pontifical Catholic University of Rio de Janeiro, Brazil
}

\begin{abstract}
The buckling of square perforated plates is analysed with the effect of shear deformation in the bending model. The relationship between the buckling load and the plate thickness is better assessed with this bending model instead of the classical model. The simply supported condition at all sides was adopted in the buckling problem such that opposite sides of the plates were uniformly compressed in one direction. The plates had a thickness to length ratio between $1 / 1000$ and $1 / 5$. This study adopted the dual reciprocity method (DRM) to obtain a formulation without domain integrals. The numerical results obtained were compared with those available in the literature.
\end{abstract}

Keywords: dual reciprocity method, plate buckling, perforated plates, Reissner plates, boundary elements.

\section{INTRODUCTION}

In-plane forces affect the bending of plates, and the reduction in plate thickness considerably strengthens the effect of the forces on the plate behavior. Buckling analysis is one of the ways to evaluate the effect of in-plane forces when the plate bending equilibrium includes the effect of geometrical non-linearity. The problem is geometrically nonlinear in the case of large deflections, i.e., the stretching and bending of the plate are coupled [1]. The buckling problem is considered when deflections remain small, and the in-plane forces are only related to in-plane tractions.

Timoshenko and Woinowsky-Krieger [4] presented the basic equation that introduces the effect of geometric non-linearity (GNL) for plate analyses using the classical bending model. Several researchers have shown that the introduction of shear deformation improves the bending model accuracy after the first studies presented by Reissner [2] and Mindlin [3]. The development was extended to bending models considering the effect of shear deformation in several studies that dealt with buckling analyses for thin or moderately thick plates [4]. Dawe and Roufaeil [5] discussed the effect of GNL in the bending of plates with shear deformation and stressed the importance of using the derivatives of rotations as well as the derivatives of deflections in the potential energy density associated with in-plane forces. Mizusawa [6] showed the effect of the derivatives of rotations was greater for certain types of boundary conditions, whereas for others, it was not significant, i.e. the derivatives of deflection would be enough.

The classical bending model has been used in the literature to perform buckling analyses of perforated plates. Levy et al. [7] studied the instability of reinforced perforated plates with a central hole under a uniform compression force. Schlack and Alois [8] computed the critical edge displacement of a simply supported pierced plate under a uniform edge displacement. Yang [9] showed that the buckling load is reduced when a square hole is considered instead of a circular hole. Brown and Yettram [10] studied how the value of the buckling parameter for different load combinations changes with the ratio between the diameter of the hole and the plate side. Shakerley and Brown [11] studied the plate buckling with eccentrically positioned holes. El-Sawy and Nazmy [12] used the finite element method to assess the 
buckling parameter value for uniaxial loaded plates with different ratios between the diameter of the hole and the plate side. The BEM formulation for plate bending including the effect of shear deformation does not require integration over the thickness, so the problem can be solved as a plane problem. An integral performed on the domain is included in the formulation to allow analyses with the GNL effect. The domain integral can be converted to one performed on the boundary with an additional numerical approach such as the multiple reciprocity method (MRM), the radial integration method (RIM) or the dual reciprocity method (DRM), which is adopted here.

The first time the name DRM was used to the conversion of the domain integral into equivalent boundary integrals was in studies on dynamic and heat transfer problems presented in Nardini and Brebbia [13]. Partridge et al. gave a more detailed explanation of the DRM in a book [14], which included some computer codes used in the method. After the first studies, the DRM has been employed in several BEM formulations including those for plate analyses with the GNL effect as summarized next. Sawaki et al. [15] studied the effect of finite displacements in the bending of thin plates. Elzein and Syngellakis [16] obtained the buckling parameters using the classical bending model. Lin et al. [17] performed studies on the buckling of rectangular and circular plates. Wang et al. [18] presented the solution for the plate-bending problem considering the effect of finite displacements using the von Kärmán equations, similarly to Wen et al. [19] who have also included the effect of shear deformation in the bending model. Purbolaksono and Aliabadi [20] presented a comparison of the results obtained with the DRM versus domain integrations to account for the GNL effect in buckling analyses using the Reissner model. Supriyono and Aliabadi [21] solved plate bending problems with the effect of the shear deformation and included effects of geometrical and physical non-linarites. Purbolaksono and Aliabadi [22] analyzed large deflections in plate bending considering the effect of shear deformation.

The BEM formulation for buckling analyses in Soares and Palermo [23] employed two integrals containing the GNL effect, with one computed in the domain and the other on the boundary. The use of first derivatives of the deflection in kernels of integrals related to the GNL effect, instead of the second derivatives, and the fact that there was no need of relating the derivatives of in-plane forces were the main features of the alternative formulation. This study employs the DRM to replace the domain integral by equivalent boundary integrals in the Soares and Palermo formulation [23]. The numerical implementation employed quadratic shape functions to approximate displacements, plate rotations, distributed shears and moments in the boundary elements as implemented in the study [23], as well as the inverse iteration and Rayleigh quotient to compute the lowest eigenvalue with the corresponding eigenvector. The changes in the value of the buckling parameter according to the plate thickness of perforated plates are studied and compared to those in the literature. The simply supported condition at all sides was adopted in the buckling problem such that opposite sides of the plates were uniformly compressed in one direction. The square plates with a central square hole had a thickness to length ratio between $1 / 1000$ and 1/5.

\section{BOUNDARY INTEGRAL EQUATIONS}

The buckling analysis considered an isotropic and homogeneous material in the perforated plate under in-plane forces distributed in the domain. The constitutive equations are written next with a unified notation for the Reissner and Mindlin bending models. The Latin indices take on values $\{1,2$ and 3$\}$ and Greek indices take on values $\{1,2\}$

$$
M_{\alpha \beta}=D \frac{(1-v)}{2}\left(u_{\alpha, \beta}+u_{\beta, \alpha}+\frac{2 v}{1-v} u_{\gamma, \gamma} \delta_{\text {á } \beta}\right)+\delta_{\alpha \beta} q R E,
$$




$$
Q_{\alpha}=D \frac{(1-v)}{2} \lambda^{2}\left(u_{\alpha}+u_{3, \alpha}\right)
$$

with

$$
\lambda^{2}=12 \frac{\kappa^{2}}{h^{2}} ; \quad R E=\frac{v}{\lambda^{2}(1-v)} .
$$

$\mathrm{D}$ is the flexural rigidity, $\mathrm{h}$ is the plate thickness, $v$ is Poisson's ratio, $\mathrm{q}$ is the distributed load on the plate domain and $\delta_{\alpha \beta}$ is the Kronecker delta. The shear parameter $\kappa^{2}$ is equal to $5 / 6$ and $\pi^{2} / 12$ for the Reissner and Mindlin models, respectively. The product qRE in eqn (2) corresponds to the linearly weighted average effect of the normal stress component in the thickness direction, which should be considered in the Reissner model [2] but not in the Mindlin model [3]. This term is null in the buckling problem because the distributed load $\mathrm{q}$ is equal to zero.

The displacement boundary integral equations (DBIEs) for the buckling problem used in Soares and Palermo [23] are given next:

$$
\begin{gathered}
\frac{1}{2} C_{i j}\left(x^{\prime}\right) u_{j}\left(x^{\prime}\right)+\int_{\Gamma}\left[T_{i j}\left(x^{\prime}, x\right) u_{j}(x)-U_{i j}\left(x^{\prime}, x\right) t_{j}(x)\right] d \Gamma(x)=\cdots \\
=\int_{\Gamma} n_{\alpha}(x) N_{\alpha \beta}(x) u_{3, \beta}(x) U_{i 3}\left(x^{\prime}, x\right) d \Gamma(x) \\
-\iint_{\Omega} N_{\alpha \beta}(X) u_{3, \beta}(X) U_{i 3, \alpha}\left(x^{\prime}, X\right) d \Omega(X)
\end{gathered}
$$

where $\mathrm{C}_{\mathrm{ij}}$ is an element of the matrix $\mathrm{C}$ related to the boundary geometry at the source point, which becomes the identity matrix when a smooth boundary is considered, $\mathrm{u}_{\alpha}$ is the plate rotation in direction $\alpha$, and $\mathrm{u}_{3}$ is the plate deflection. $U_{\mathrm{ij}}$ represents the rotation $(\mathrm{j}=1,2)$ or the deflection $(\mathrm{j}=3)$ due to a unit couple $(\mathrm{i}=1,2)$ or a unit point force $(\mathrm{i}=3) . \mathrm{T}_{\mathrm{ij}}$ represents the moment $(j=1,2)$ or the shear $(j=3)$ due to a unit couple $(i=1,2)$ or a unit point force $(i=3) . U_{i j}$ and $\mathrm{T}_{\mathrm{ij}}$ are related to the fundamental solution.

According to the development in Soares and Palermo [23], the GNL effect in eqn (3) is introduced with an integral performed on the domain and a boundary integral, which is related to the natural condition in the part of the boundary when the deflection $\left(u_{3}\right)$ is not prescribed. The result in eqn (17) Soares and Palermo [23] showed the boundary integral containing the GNL effect should only be computed along the boundary part with the prescribed deflection. The GNL effect requires the use of the BIE for the gradient of the deflection, which is written next for an internal collocation point:

$$
\begin{aligned}
u_{3, \gamma}\left(X^{\prime}\right)= & \int_{\Gamma}\left\{n_{\alpha}(x) M_{3 \alpha \beta, \gamma}\left(X^{\prime}, x\right) u_{\beta}(x)+n_{\beta}(x) Q_{3 \beta, \gamma}\left(X^{\prime}, x\right) u_{3}(x)+\cdots\right. \\
& \left.\ldots-U_{3 \beta, \gamma}\left(X^{\prime}, x\right) t_{\beta}(x)-U_{33, \gamma}\left(X^{\prime}, x\right) t_{3}(x)\right\} d \Gamma(x) \\
& -\int_{\Gamma} n_{\alpha}(x) N_{\alpha \beta}(x) u_{3, \beta}(x) U_{i 3, \gamma}\left(X^{\prime}, x\right) d \Gamma(x)+\cdots \\
& \ldots+\iint_{\Omega} N_{\alpha \beta}(X) u_{3, \beta}(X) U_{33, \alpha \gamma}\left(X^{\prime}, X\right) d \Omega(X) .
\end{aligned}
$$


Eqn (4) was written with kernels differentiated with respect to the field point coordinates. The tangential differential operator can be introduced in kernels of integrals in eqn (4) to reduce the singularities resulting from the differentiation as shown in Palermo [24].

The generalized plane stress problem is solved once to obtain the in-plane force distribution used in the buckling analysis. The in-plane force distribution is dependent of the shape and the dimensions of the hole in perforated plates beyond the in-plane tractions distribution on the boundary.

\section{APPLICATION OF THE DUAL RECIPROCITY METHOD}

The kernel in the domain integral of the DBIEs (eqn (3)) contains the product between the gradient of deflection from the fundamental solution, the in-plane forces tensor and the gradient of the plate deflection. A vector function (b) resulting from the product between the in-plane forces tensor and the gradient of plate deflection can be defined, i.e.:

$$
b_{\theta}(X)=N_{\theta \beta}(X) u_{3, \beta}(X) .
$$

The DRM is introduced in the approximation of the vector function (b) using the following relation [14]:

$$
b_{\theta}(X)=\sum_{m=1}^{N+L} \alpha_{\theta}^{m} f^{m} .
$$

The summation in eqn (6) is extended along all points employed in the DRM, i.e. the total number of points placed on the boundary $(\mathrm{N})$ and in the domain $(\mathrm{L}), \mathrm{f}^{\mathrm{m}}$ and $\alpha^{\mathrm{m}}$ are sets of the approximating functions and weighting coefficients, respectively [14]. Furthermore, the application of the DRM employs the particular solution $\hat{\mathrm{u}}_{\mathrm{j}}^{\mathrm{m}}[$ [14], which is related to the approximating function $\mathrm{f}^{\mathrm{m}}$ according to following equations:

$$
\begin{gathered}
\nabla^{2}\left(\nabla^{2} \phi\right)=\frac{1}{D} f(r) . \\
\nabla^{2}\left(\hat{\mathrm{u}}_{3}\right)=\frac{2}{D(1-v) \lambda^{2}} f(r)-\nabla^{2} \phi . \\
\hat{\mathrm{u}}_{\alpha}=\phi_{, \alpha} .
\end{gathered}
$$

Eqns (7) to (9) are similar to those used to obtain the fundamental solution for the unit point load under the static condition in Palermo [25] and the dynamic condition in Palermo [26]. The well-known radial basis function $(1+\mathrm{r})$ is considered for $\mathrm{f}^{\mathrm{m}}$. The corresponding particular solution, which is similar to that presented in Wen et al. [27], is given by:

$$
f^{m}=1+r \Rightarrow\left\{\begin{array}{l}
\hat{\mathrm{u}}_{\alpha}^{m}=\frac{1}{D}\left(\frac{\mathrm{r}^{3}}{16}+\frac{\mathrm{r}^{4}}{45}\right) r_{,}, \\
\hat{\mathrm{u}}_{3}^{m}=\frac{2}{D(1-v) \lambda^{2}}\left(\frac{\mathrm{r}^{2}}{4}+\frac{\mathrm{r}^{3}}{9}\right)-\frac{1}{D}\left(\frac{\mathrm{r}^{4}}{64}+\frac{\mathrm{r}^{5}}{225}\right) .
\end{array}\right.
$$

The distributed shear and bending moments related to the particular solutions are obtained with the constitutive eqns (1) and (2). The generalized tractions are obtained from the distributed shear and bending moments using the following relations:

$$
\begin{aligned}
& \hat{t}_{\propto}^{m}=\widehat{M}_{\propto \beta}^{m} n_{\beta} \\
& \hat{t}_{3}^{m}=\widehat{Q}_{\beta}^{m} n_{\beta} .
\end{aligned}
$$

The gradient of the deflection $\left(U_{i 3, \alpha}\right)$ from the fundamental solution multiplies the terms related to the GNL effect in the DBIEs (eqn (3)). The introduction of the DRM to replace the domain integral requires the use of the BIE for the gradient of the deflections. This equation 
is written next with the kernel of the domain integral containing the function $\mathrm{f}^{\mathrm{m}}$ and the boundary integrals using the displacements and tractions related to the particular solution $\hat{\mathrm{u}}_{\mathrm{j}}^{\mathrm{m}}$ [14]:

$$
\begin{array}{r}
c \hat{u}_{i, \theta}^{m}\left(x^{\prime}\right)=\int_{\Gamma}\left[n_{\alpha}(x) M_{i \alpha \beta, \theta}\left(x^{\prime}, x\right) \hat{u}_{\beta}^{m}(x)+n_{\beta}(x) Q_{i \beta, \theta}\left(x^{\prime}, x\right) \hat{u}_{3}^{m}(x)+\cdots\right. \\
\left.\ldots-U_{i \beta, \theta}\left(x^{\prime}, x\right) \hat{t}_{\beta}^{m}(x)-U_{i 3, \theta}\left(x^{\prime}, x\right) \hat{t}_{3}^{m}(x)\right] d \Gamma(x)-\iint_{\Omega} f^{m}(X) U_{i 3, \theta}\left(x^{\prime}, X\right) d \Omega(X) .
\end{array}
$$

Eqn (12) was written with kernels differentiated with respect to the field coordinates, the scalar $\mathrm{c}$ is equal to 1 in case of collocation points located inside the domain and 0.5 in case of points placed on smooth parts of the boundary. The continuity of the first derivative of displacements at $x^{\prime}$ is required to apply eqn (12) at points on the boundary. The DBIE for the buckling problem using eqn (12) to introduce the DRM is given by

$$
\begin{gathered}
\quad \frac{1}{2} C_{i j}\left(x^{\prime}\right) u_{j}\left(x^{\prime}\right)+\int_{\Gamma}\left[T_{i j}\left(x^{\prime}, x\right) u_{j}(x)-U_{i j}\left(x^{\prime}, x\right) t_{j}(x)\right] d \Gamma(x)=\cdots \\
=\int_{\Gamma} n_{\alpha}(x) N_{\alpha \beta}(x) u_{3, \beta}(x) U_{i 3}\left(x^{\prime}, x\right) d \Gamma(x)+\sum_{m=1}^{N+L} \alpha_{\theta}^{m}\left\{c \hat{u}_{i, \theta}^{m}\left(x^{\prime}\right)+\cdots\right. \\
\ldots \ldots .-\int_{\Gamma}\left[n_{\alpha}(x) M_{i \alpha \beta, \theta}\left(x^{\prime}, x\right) \hat{u}_{\beta}^{m}(x)+n_{\beta}(x) Q_{i \beta, \theta}\left(x^{\prime}, x\right) \hat{u}_{3}^{m}(x)+\cdots\right. \\
\left.\left.\ldots-U_{i \beta, \theta}\left(x^{\prime}, x\right) \hat{t}_{\beta}^{m}(x)-U_{i 3, \theta}\left(x^{\prime}, x\right) \hat{t}_{3}^{m}(x)\right] d \Gamma(x)\right\} .
\end{gathered}
$$

The second derivatives of the deflection $\left(\mathrm{U}_{33, \alpha \gamma}\right)$ of the fundamental solution multiply the terms related to the GNL effect in the BIE for the gradient of the deflection (eqn (4)). The introduction of the DRM also requires the BIE for the second derivative of the deflection, as explained to obtain eqn (13). The BIE for the second derivative of the deflection with the kernel of the domain integral containing $\mathrm{f}^{\mathrm{m}}$ is given by:

$$
\begin{gathered}
c \hat{u}_{3, \gamma \theta}^{m}\left(x^{\prime}\right)+\int_{\Gamma}\left[n_{\alpha}(x) M_{3 \alpha \beta, \gamma \theta}\left(x^{\prime}, x\right) \hat{u}_{\beta}^{m}(x)+n_{\beta}(x) Q_{3 \beta, \gamma \theta}\left(x^{\prime}, x\right) \hat{u}_{3}^{m}(x)+\cdots\right. \\
\left.\ldots-U_{3 \beta, \gamma \theta}\left(x^{\prime}, x\right) \hat{t}_{\beta}^{m}(x)-U_{33, \gamma \theta}\left(x^{\prime}, x\right) \hat{t}_{3}^{m}(x)\right] d \Gamma(x) \\
=\iint_{\Omega} f^{m}(X) U_{33, \gamma \theta}\left(x^{\prime}, X\right) d \Omega(X) .
\end{gathered}
$$

Eqn (14) was written with kernels differentiated with respect to the field coordinates, the scalar $\mathrm{c}$ is equal to 1 in case of collocation points located inside the domain and 0.5 in case of points placed on smooth parts of the boundary. The continuity of the second derivative of the deflection at $x^{`}$ is required to apply eqn (14) at points on the boundary. The BIE for the second derivative of the deflection using eqn (14) to introduce the DRM is given by.

$$
\begin{gathered}
g u_{3, \gamma}\left(x^{\prime}\right)=\int_{\Gamma}\left\{n_{\alpha}(x) M_{3 \alpha \beta, \gamma}\left(x^{\prime}, x\right) u_{\beta}(x)+n_{\beta}(x) Q_{3 \beta, \gamma}\left(x^{\prime}, x\right) u_{3}(x)+\cdots\right. \\
\left.\ldots-U_{3 \beta, \gamma}\left(x^{\prime}, x\right) t_{\beta}(x)-U_{33, \gamma}\left(x^{\prime}, x\right) t_{3}(x)\right\} d \Gamma(x) \\
-\int_{\Gamma} n_{\alpha}(x) N_{\alpha \beta}(x) u_{3, \beta}(x) U_{i 3, \gamma}\left(x^{\prime}, x\right) d \Gamma(x)+\cdots \\
\ldots+\sum_{m=1}^{N+L} \alpha_{\theta}^{m}\left\{\begin{array}{c}
c \hat{u}_{i, \gamma \theta}^{m}\left(x^{\prime}\right)+\int_{\Gamma}\left[n_{\alpha}(x) M_{i \alpha \beta, \gamma \theta}\left(x^{\prime}, x\right) \hat{u}_{\beta}^{m}(x)+n_{\beta}(x) Q_{i \beta, \gamma \theta}\left(x^{\prime}, x\right) \hat{u}_{3}^{m}(x)+\ldots\right. \\
\left.\left.\ldots-U_{i \beta, \gamma \theta}\left(x^{\prime}, x\right) \hat{t}_{\beta}^{m}(x)-U_{i 3, \gamma \theta}\left(x^{\prime}, x\right) \hat{t}_{3}^{m}(x)\right] d \Gamma(x)\right\} .
\end{array}\right.
\end{gathered}
$$


The scalar $g$ in eqn (15) has the same values explained to the scalar c in eqns (12) and (14).

\section{NUMERICAL IMPLEMENTATION}

The discretization of the BIEs employed quadratic isoparametric boundary elements and the collocation points were always placed on the boundary. The same mapping function was used for conformal and non-conformal interpolations. The collocation points were placed at positions $(-0.67$ and 0.0$)$, in the range $(1,1)$, in the case of continuous elements and at positions $(-0.67,0.0,+0.67)$ in the case of discontinuous elements, i.e. the collocation points were always shifted inside the boundary elements. The singularity subtraction [28] and the transformation of variable technique [29] were employed for the Cauchy and weak-type singularities, respectively, when integrations were performed on elements containing the collocation points. Hyper- and super-singular integrals in elements containing the collocation points were numerically evaluated using the computer code presented in Gao [30]. The standard Gauss-Legendre scheme was employed for integrations on elements not containing the collocation points.

The DRM considered points distributed in the domain and on the boundary. The points on the boundary were placed at the positions of the collocation points. The first boundary integral on the left-hand side (LHS) of eqn (13) is not related with the DRM, as well as the second boundary integral on the LHS of eqn (15). The discretization of those boundary integrals assumed a constant value along each boundary element for the sum of products between the gradient of the deflection and the in-plane forces, which values were obtained at the central node. The values of derivatives of the deflection computed at the central node used by the DRM were also used in the computation of those boundary integrals.

The eigenvalue analysis used the basic inverse iteration with the Rayleigh quotient [31] as employed in Soares and Palermo [23] and written next:

$$
\begin{gathered}
A x^{(k+1)}=B x^{k}, \\
\lambda_{k}=\frac{\left(x^{(k+1)}, x^{k}\right)}{\left(x^{(k+1)}, x^{(k+1)}\right)} .
\end{gathered}
$$

The vector $\mathrm{x}^{\mathrm{k}}$ in eqns (16) to (17) is related to values of the gradient of the deflection at the DRM points. The discretized forms of eqns (13) and (15) were used instead of eqn (16) as done in Soares and Palermo [23]. Starting with an eigenvector $\mathrm{x}^{1}$ with elements equal to 1.0 , the values of the displacements and tractions at the boundary nodes are found with eqn (13); these values are introduced in eqn (15) to obtain the gradient of the deflection (elements of the eigenvector $\mathrm{x}^{2}$ ). The elements of $\alpha$ are recalculated at each iteration step according to values of the gradient of the deflection introduced in eqn (5).

After the computation of elements $b_{\theta}$, the solution of the system of equations given by eqn (6) carries the values of $\alpha_{\theta}{ }^{\mathrm{m}}$ as explained in detail in Partridge et al. [14]. The index $\theta$ in elements of $b$ and $\alpha$ requires eqns (5) and (6) to be used twice and one set of $\alpha$ is obtained for each direction $\theta$ of the plate.

\section{NUMERICAL EXAMPLES}

The perforated plate shown in Fig. 1 was studied in El-Sawy and Nazmy [12] where a correction was suggested for the buckling parameters presented in Sabir and Chow [32] for cases of large dimension holes. The results obtained in Soares and Palermo [23] for this problem were very close to those in El-Sawy and Nazmy [12] and were considered here to evaluate the application of the DRM. 


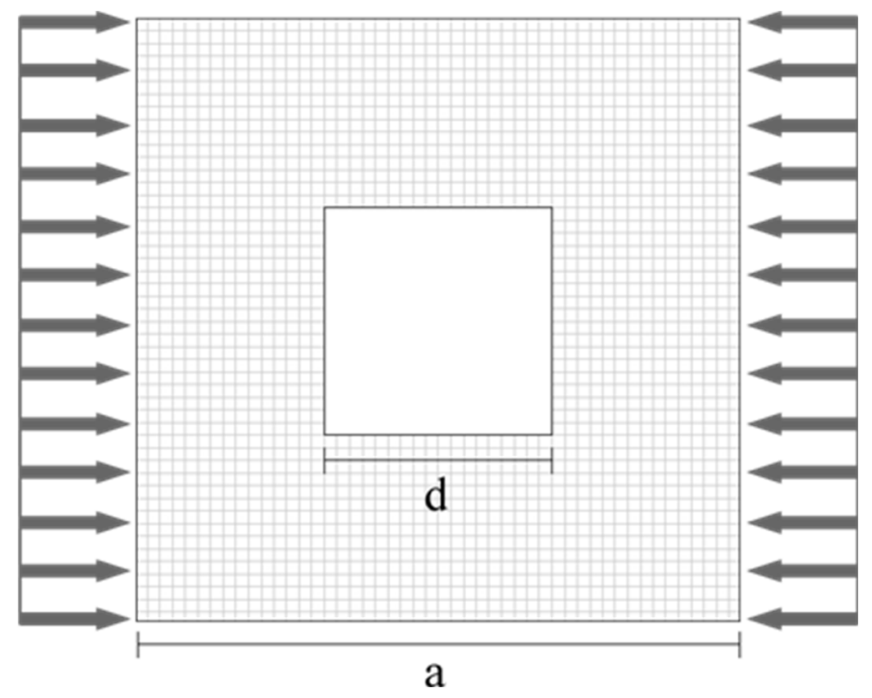

Figure 1: Square plate containing a square hole at the center.

The buckling parameter $\mathrm{k}$ is a non-dimensional value related to the critical load of the plate $\left(\mathrm{N}_{\mathrm{cr}}\right)$, the length of the plate side (a) and the flexural rigidity (D), which is obtained according to following expression:

$$
k=\frac{a^{2} N_{c r}}{\pi^{2} D} .
$$

The simply supported condition at all sides was adopted in the buckling problem such that opposite sides of the plates were uniformly compressed in one direction. The plates had a thickness to length ratio between $1 / 1000$ and $1 / 5$. The normalized square hole dimensions $(\mathrm{d} / \mathrm{a})$ were in the range 0.1 to 0.7 . The Young's modulus (E) and Poisson's ratio (v) were $206.9 \mathrm{GPa}$ and 0.3 , respectively. The value of the shear parameter $\kappa^{2}$ was $\pi^{2} / 12$. The comparison between values for the buckling parameter obtained with the DRM and those presented in [23], in which the GNL effect was introduced with domain integration using cells, are presented in Tables 1 to 5.

Table 1: Critical loads for the perforated plate obtained with DRM.

\begin{tabular}{|c|c|c|c|c|c|}
\hline \multirow{2}{*}{$\begin{array}{c}\text { Boundary } \\
\text { elements }\end{array}$} & \multirow{2}{*}{$\begin{array}{c}\text { Domain } \\
\text { points }\end{array}$} & \multirow{2}{*}{$\mathrm{d} / \mathrm{a}$} & \multicolumn{3}{|c|}{$\mathrm{h} / \mathrm{a}=0.001$} \\
\cline { 4 - 6 } & & $\mathrm{DRM}$ & {$[23]$} & Diff. (\%) \\
\hline 480 & 1564 & 0.1 & 3.7975 & 3.7994 & -0.05 \\
\hline 480 & 1500 & 0.2 & 3.4442 & 3.4494 & -0.15 \\
\hline 480 & 1568 & 0.3 & 3.1854 & 3.1883 & -0.09 \\
\hline 480 & 1536 & 0.4 & 3.0194 & 3.0297 & -0.34 \\
\hline 480 & 1533 & 0.5 & 2.9066 & 2.9310 & -0.84 \\
\hline 480 & 1548 & 0.6 & 2.8431 & 2.8664 & -0.82 \\
\hline 1280 & 1632 & 0.7 & $2.8168^{*}$ & 2.8449 & -1.00 \\
\hline
\end{tabular}


Table 2: Critical loads for the perforated plate obtained with DRM.

\begin{tabular}{|c|c|c|c|c|c|}
\hline \multirow{2}{*}{$\begin{array}{c}\text { Boundary } \\
\text { elements }\end{array}$} & \multirow{2}{*}{$\begin{array}{c}\text { Domain } \\
\text { points }\end{array}$} & $\mathrm{d} / \mathrm{a}$ & \multicolumn{3}{|c|}{$\mathrm{h} / \mathrm{a}=0.01$} \\
\cline { 4 - 6 } & & & $\mathrm{DRM}$ & {$[23]$} & Diff. (\%) \\
\hline 480 & 1564 & 0.1 & 3.7909 & 3.7932 & -0.06 \\
\hline 480 & 1500 & 0.2 & 3.4343 & 3.4412 & -0.20 \\
\hline 480 & 1568 & 0.3 & 3.1723 & 3.1782 & -0.18 \\
\hline 480 & 1536 & 0.4 & 3.0012 & 3.0153 & -0.47 \\
\hline 480 & 1533 & 0.5 & 2.8879 & 2.9096 & -0.75 \\
\hline 480 & 1548 & 0.6 & 2.8198 & 2.8352 & -0.55 \\
\hline 480 & 1632 & 0.7 & 2.8131 & 2.7998 & 0.47 \\
\hline
\end{tabular}

Table 3: Critical loads for the perforated plate obtained with DRM.

\begin{tabular}{|c|c|c|c|c|c|}
\hline \multirow{2}{*}{$\begin{array}{c}\text { Boundary } \\
\text { elements }\end{array}$} & \multirow{2}{*}{$\begin{array}{c}\text { Domain } \\
\text { points }\end{array}$} & $\mathrm{d} / \mathrm{a}$ & \multicolumn{3}{|c|}{$\mathrm{h} / \mathrm{a}=0.05$} \\
\cline { 4 - 6 } & & & $\mathrm{DRM}$ & {$[23]$} & Diff. (\%) \\
\hline 480 & 1564 & 0.1 & 3.7284 & 3.7313 & -0.08 \\
\hline 480 & 1500 & 0.2 & 3.3659 & 3.3747 & -0.26 \\
\hline 480 & 1568 & 0.3 & 3.0957 & 3.1034 & -0.25 \\
\hline 480 & 1536 & 0.4 & 2.9028 & 2.9207 & -0.62 \\
\hline 480 & 1533 & 0.5 & 2.7565 & 2.7820 & -0.92 \\
\hline 480 & 1548 & 0.6 & 2.6412 & 2.6625 & -0.81 \\
\hline 480 & 1632 & 0.7 & 2.5730 & 2.5629 & 0.39 \\
\hline
\end{tabular}

Table 4: Critical loads for the perforated plate obtained with DRM.

\begin{tabular}{|c|c|c|c|c|c|}
\hline \multirow{2}{*}{$\begin{array}{c}\text { Boundary } \\
\text { elements }\end{array}$} & \multirow{2}{*}{$\begin{array}{c}\text { Domain } \\
\text { points }\end{array}$} & $\mathrm{d} / \mathrm{a}$ & \multicolumn{3}{|c|}{$\mathrm{h} / \mathrm{a}=0.10$} \\
\cline { 4 - 6 } & & & $\mathrm{DRM}$ & {$[23]$} & Diff. (\%) \\
\hline 480 & 1564 & 0.1 & 3.5780 & 3.5819 & -0.11 \\
\hline 480 & 1500 & 0.2 & 3.2222 & 3.2344 & -0.38 \\
\hline 480 & 1568 & 0.3 & 2.9471 & 2.9577 & -0.36 \\
\hline 480 & 1536 & 0.4 & 2.7247 & 2.7495 & -0.91 \\
\hline 480 & 1533 & 0.5 & 2.5346 & 2.5670 & -1.28 \\
\hline 480 & 1548 & 0.6 & 2.3614 & 2.3884 & -1.14 \\
\hline 480 & 1632 & 0.7 & 2.2611 & 2.2716 & -0.47 \\
\hline
\end{tabular}


Table 5: Critical loads for the perforated plate obtained with DRM.

\begin{tabular}{|c|c|c|c|c|c|}
\hline \multirow{2}{*}{$\begin{array}{c}\text { Boundary } \\
\text { elements }\end{array}$} & \multirow{2}{*}{$\begin{array}{c}\text { Domain } \\
\text { points }\end{array}$} & \multirow{2}{*}{$\mathrm{d} / \mathrm{a}$} & \multicolumn{3}{|c|}{$\mathrm{h} / \mathrm{a}=0.20$} \\
\cline { 4 - 6 } & & & $\mathrm{DRM}$ & {$[23]$} & Diff. (\%) \\
\hline 480 & 1564 & 0.1 & 3.0798 & 3.1043 & -0.79 \\
\hline 480 & 1500 & 0.2 & 2.7555 & 2.8052 & -1.81 \\
\hline 480 & 1568 & 0.3 & 2.4799 & 2.5138 & -1.37 \\
\hline 480 & 1536 & 0.4 & 2.2025 & 2.2235 & -0.95 \\
\hline 1280 & 1533 & 0.5 & $1.6960^{*}$ & 1.7185 & -1.33 \\
\hline 1280 & 1548 & 0.6 & $1.2087^{*}$ & 1.1755 & 2.75 \\
\hline 1280 & 1632 & 0.7 & $0.7813^{*}$ & 0.7263 & 7.04 \\
\hline
\end{tabular}

\section{CONCLUSIONS}

The results obtained with the application of the DRM in the formulation presented in Soares and Palermo [23] were close to the solution with the domain integration using cells. Most solutions employed 480 quadratic boundary elements ( 384 elements on the external boundary and 96 elements along the boundary of the hole) while a few cases required 1280 quadratic boundary elements (1024 elements on the external boundary and 256 elements along the boundary of the hole). A simple radial basis function $(1+\mathrm{r})$ for $\mathrm{f}^{\mathrm{m}}$ was used and no shape parameter was adopted. No additional tools were employed to improve the convergence or the integrations over the boundary elements in the numerical implementation of the DRM. The authors believe this DRM formulation for buckling analysis presented accurate results and a consistent behavior with reference to that shown in Soares and Palermo [23].

The differences in the variation of the buckling parameter according to the thickness to plate side ratio shown in Fig. 2 could only be identified when the effect of shear deformation was included in the bending model. The next step in the study of the buckling of perforated plates will be the analysis of circular holes in square and rectangular perforated plates, which will be presented soon.

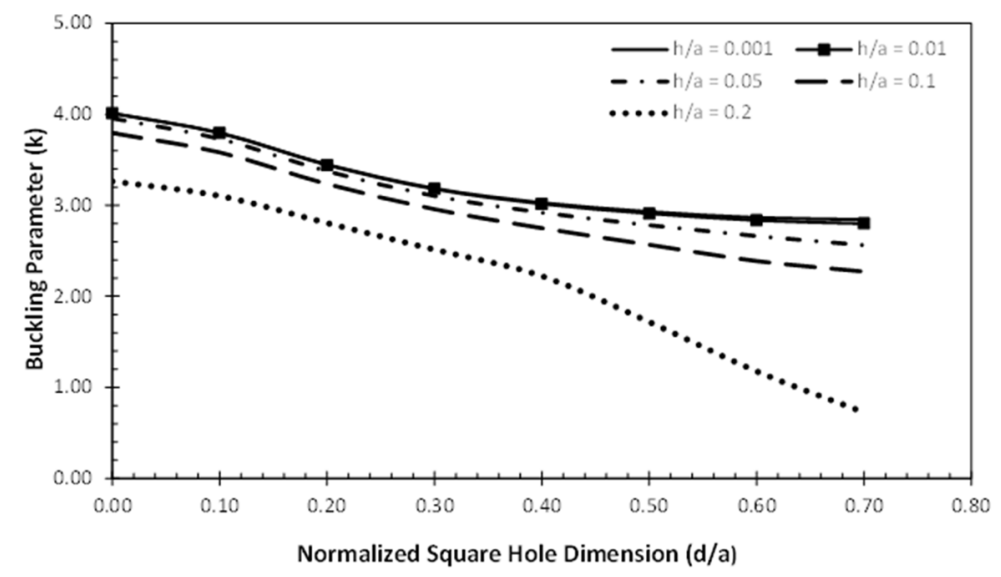

Figure 2: The effect of the hole dimension on the value of the buckling parameter. 


\section{ACKNOWLEDGEMENTS}

The authors are grateful to CNPq (305997/2016-5) and CAPES (01P03713/2017) for their support in the development of this research.

\section{REFERENCES}

[1] Palermo Jr., L., Simões, R. \& Mesquita Neto, E., Finite Displacement Analysis of Plates Subjected to Flexure and Compression Using the Boundary Element Method, International Colloquium on Stability and Ductility of Steel Structures, Lisboa, 2006.

[2] Reissner, E., The effect of transverse shear deformation on the bending of elastic plates. Journal of Applied Mechanics, 12(2), pp. A66-A77, 1945.

[3] Mindlin, R.D., Influence of rotatory inertia and shear on flexural motions of isotropic elastic plates. Journal of Applied Mechanics, 18, pp. 18-31, 1951.

[4] Timoshenko, S.P. \& Woinowsky-Krieger, S., Theory of Plates and Shells, McGrawHill Book Company: New York, 2nd ed., 1959.

[5] Dawe, D.J. \& Roufaeil, O.L., Buckling of rectangular Mindlin plates. Computers \& Structures, 15(4), pp. 461-471, 1982.

[6] Mizusawa, T., Buckling of rectangular Mindlin plates with tapered thickness by the spline strip method. International Journal of Solids \& Structures, 30(12), pp. 16631677, 1993.

[7] Levy, S., Woolley, R.M. \& Kroll, W.D., Instability of simply supported square plate with reinforced circular hole in edge compression. Journal of Research, pp. 571-577, 1947.

[8] Schlack, J.R. \& Alois, L., Elastic stability of pierced square plates. Experimental Mechanics, 4(6), pp. 167-172, 1964.

[9] Yang, H.T., A Finite Element Formulation for Stability Analysis of Doubly Curved Thin Shell Structures. PhD thesis, Cornell University, 1969.

[10] Brown, C.J. \& Yettram, A.L., The elastic stability of square perforated plates under combinations of bending, shear and direct load. Thin-Walled Structures, 4(3), pp. 239246, 1986.

[11] Shakerley, T.M. \& Brown, C.J., Elastic buckling of plates with eccentrically positioned rectangular perforations. International Journal of Mechanical Sciences, 38(8), pp. 825-838, 1996.

[12] El-Sawy, K.M. \& Nazmy, A.S., Effect of aspect ratio on the elastic buckling of uniaxially loaded plates with eccentric holes. Thin-Walled Structures, 39(12), pp. 983998, 2001.

[13] Nardini, D. \& Brebbia, C.A., Boundary integral formulation of mass matrices for dynamic analysis. Time-dependent and Vibration Problems, Springer: Berlin and Heidelberg, pp. 191-208, 1985.

[14] Partridge, P.W., Brebbia, C.A. \& Wrobel, L.C., The Dual Reciprocity Boundary Element Method, Computational Mechanics Publication and Elsevier Applied Science: London and New York, 1992.

[15] Sawaki, Y., Takeuchi, K. \& Kamiya, N., Nonlinear bending analysis without domaincell discretisation. Engineering Analysis with Boundary Elements, 7(3), pp. 130-135, 1990.

[16] Elzein, A. \& Syngellakis, S., The dual reciprocity in boundary element formulations of the plate buckling problem. Engineering Analysis with Boundary Elements, 9(2), pp. 175-184, 1992. 
[17] Lin, J., Duffield, R.C. \& Shih, H., Buckling analysis of elastic plates by boundary element method. Engineering Analysis with Boundary Elements, 23(2), pp. 131-137, 1999.

[18] Wang, W., Ji, X. \& Tanaka, M., A dual reciprocity boundary element approach for the problems of large deflection of thin elastic plates. Computational Mechanics, 26(1), pp. 58-65, 2000

[19] Wen, P.H., Aliabadi, M.H. \& Young, A., Large deflection analysis of Reissner plate by boundary element method. Computers \& Structures, 83(10-11), pp. 870-879, 2005.

[20] Purbolaksono, J. \& Aliabadi, M.H., Large deformation of shear deformable plate by boundary element method. Journal of Engineering Mathematics, 51, pp. 211-230, 2005.

[21] Supriyono \& Aliabadi, M.H., Analysis of shear deformable plates with combined geometric and material nonlinearities by boundary element method. International Journal of Solids and Structures, 44(3-4), pp. 1038-1059, 2007.

[22] Purbolaksono, J. \& Aliabadi, M.H., Stability of Euler's method for evaluating large deformation of shear deformable plates by dual reciprocity boundary element method. Engineering Analysis with Boundary Elements, 34(9), pp. 819-823, 2010.

[23] Soares Jr., R.A. \& Palermo Jr., L., Effect of shear deformation on the buckling parameter of perforated and non-perforated plates studied using the boundary element method. Engineering Analysis with Boundary Elements, 85, pp. 57-69, 2017.

[24] Palermo Jr., L., The tangential differential operator applied to a stress boundary integral equation for plate bending including the shear deformation effect. Engineering Analysis with Boundary Elements, 36, pp. 1213-1225, 2012.

[25] Palermo Jr., L., Plate bending analysis using the classical or the Reissner-Mindlin models. Engineering Analysis with Boundary Elements, 27, pp. 603-609, 2003.

[26] Palermo Jr., L., On the harmonic response of plates with the shear deformation effect using the elastodynamic solution in the boundary element method. Engineering Analysis with Boundary Elements, 31, pp. 176-183, 2007.

[27] Wen, P.H., Aliabadi, M.H. \& Young, A., Application of dual reciprocity method to plates and shells. Engineering Analysis with Boundary Elements, 24(7-8), pp. 583590,2000

[28] Aliabadi, M.H., The Boundary Element Method: Applications in Solids and Structures, John Wiley \& Sons Ltd., 2002.

[29] Telles, J.C.F., A self-adaptive coordinate transformation for efficient numerical evaluation of general boundary element integrals. International Journal for Numerical Methods in Engineering, 24, pp. 959-973, 1987.

[30] Gao, X., Numerical evaluation of two-dimensional singular boundary integrals Theory and Fortran code. Journal of Computational and Applied Mathematics, 188, pp. 44-64, 2006.

[31] Wilkinson, J.H., The Algebraic Eigenvalue Problem, Oxford University Press and William Clowes \& Sons: London, 1972.

[32] Sabir, A.B. \& Chow, F.Y., Elastic buckling of flat panels containing circular and square holes. Proceedings of the International Conference on Instability and Plastic Collapse of Steel Structures, ed. L.J. Morris, Granada Publishing: London, pp. 31121, 1983. 